\title{
Homo-harringtonine (HHT) - A highly effective drug against coronaviruses and the potential for large-scale clinical applications
}

\author{
Hai-Jun $\mathrm{Wen}^{1 \dagger}$, Pei $\operatorname{Lin}^{1 \dagger}$, Gong-Xun Zhong ${ }^{2,3 \dagger}$, Zhi-Chao Xu' ${ }^{1}$, Lei Shuai ${ }^{2,3}$, Zhi-Yuan \\ $\mathrm{Wen}^{2,3}$, Chong Wang ${ }^{2,3}$, Xue $\mathrm{Cao}^{4}$, Wen-Bin $\mathrm{He}^{5,6}$, Jing Feng ${ }^{5,6}$, Qi-Chun Cai ${ }^{7}$, Hua-Juan \\ $\mathrm{Ma}^{7}$, Si-Jin $\mathrm{Wu}^{8}$, Guo-Dong Wang ${ }^{5,6}$, Xue-Mei Lyu ${ }^{5,6}$, Feng-Liang Liu ${ }^{9,10}$, Yong-Tang \\ Zheng $^{9,10}$, Hui Zeng ${ }^{11}$, Xiong-Lei He ${ }^{1}$, Hualan Chen ${ }^{2,3} *$, Fu-Jie Zhang ${ }^{11}$, and Chung-I \\ $\mathrm{Wu}^{1,12 *}$
}

1. State Key Laboratory of Biocontrol, School of Life Sciences, Sun Yat-sen University, Guangzhou 510275, China.

2. State Key Laboratory of Veterinary Biotechnology, Harbin Veterinary Researched Institute, Chinese Academy of Agricultural Sciences, Harbin 150069, People's Republic of China.

3. National High Containment Laboratory for Animal Diseases Control and Prevention, Harbin 150069, People's Republic of China.

4. Department of Laboratory Animal Science, Kunming Medical University, Kunming 650500, People's Republic of China.

5. State Key Laboratory of Genetic Resources and Evolution, Kunming Institute of Zoology, Chinese Academy of Science, Kunming 650223, China.

6. Center for Excellence in Animal Evolution and Genetics, Chinese Academy of Sciences, Kunming 650223, China.

7. Cancer Center, Clifford Hospital, Jinan University, Guangzhou, People's Republic of China.

8. Laboratory of Molecular Modeling and Design, State Key Laboratory of Molecular Reaction

Dynamics, Dalian Institute of Chemical Physics, Chinese Academy of Sciences, Dalian 116024, China

9. Key Laboratory of Animal Models and Human Disease Mechanisms of Chinese Academy of Sciences / Key Laboratory of Bioactive Peptides of Yunnan Province, Kunming Institute of Zoology, Chinese Academy of Sciences, Kunming, Yunnan 650223, China.

10. Kunming National High-level Bio-safety Research Center for Non-human Primates, Center for Biosafety Mega-Science, Kunming Institute of Zoology, Chinese Academy of Sciences, Kunming, 650107, China.

11. Beijing Ditan Hospital, Capital Medical University, Beijing 100102, China.

12. Southern Marine Science and Engineering Guangdong Laboratory (Zhuhai), Zhuhai 519082, People's Republic of China.

$\dagger$ These authors contribute equally to this work.

*Correspondence to:

Chung-I Wu, wzhongyi@mail.sysu.edu.cn

Fu-Jie Zhang, treatment@chinaaids.cn

Hua-Lan Chen, chenhualan@caas.cn 


\begin{abstract}
In the search for treatment schemes of COVID-19, we start by examining the general weakness of coronaviruses and then identify approved drugs attacking that weakness. The approach, if successful, should identify drugs with a specific mechanism that is at least as effective as the best drugs proposed and are ready for clinical trials. All coronaviruses translate their non-structural proteins $(\sim 16)$ in concatenation, resulting in a very large super-protein. Homo-harringtonine (HHT), which has been approved for the treatment of leukemia, blocks protein elongation very effectively. Hence, HHT can repress the replication of many coronaviruses at the nano-molar concentration. In two mouse models, HHT clears SARS-CoV-2 in 3 days, especially by nasal dripping of $40 \mathrm{ug}$ per day. We also use dogs to confirm the safety of HHT delivered by nebulization. The nebulization scheme could be ready for large-scale applications at the onset of the next epidemics. For the current COVID-19, a clinical trial has been approved by the Ditan hospital of Beijing but could not be implemented for want of patients. The protocol is available to qualified medical facilities.
\end{abstract}

\title{
Introduction
}

Coronaviruses have increasingly become the causal agent of epidemics in humans as well as in domesticated animals $(1,2)$. Since the beginning of this century, there have been 3 such epidemics, SARS (2003), MERS (2012) and COVID-19, and they may not be the last ones $(1,3)$. At present, there is no highly effective and broadly applicable treatment scheme to thwart the COVID-19 pandemic. Obviously, an effective treatment scheme should be desirable. Although such a scheme may or may not be in time for this current pandemic, it can certainly be ready as the first-line defense at the onset of the next coronavirus epidemics.

This current report differs from other proposed schemes for treating COVID-19 (see the next section) in that we start by identifying the general weakness of the coronaviruses and then search for approved drugs that target the viruses by a specific mechanism. In contrast, the mainstream approach is to screen for effective drugs and then identify the repression mechanisms. There is a three-fold advantage to our approach if (and only if) the weakness of the viruses and the drug targeting this weakness can be identified. First, many of the experiments would have been done and published. Second, the efficacy of the drug can be set at a level higher than the most effective agents proposed to treat the infection. Third, since the scheme would target coronaviruses in general, it should be ready at the onset of the next epidemics caused by coronaviruses.

\section{A brief survey of drugs proposed for treating COVID-19}


To have an overview of the proposals for treating COVID-19, we carry out a survey of drugs targeting the virus itself. The survey is of a limited scope as schemes that boost or suppress the immunity against the virus (such as cytokine storms) are not included. In particular, we focus on the schemes that target the cellular components involved in the replication of SARSCoV-2. Presumably, direct attacks on the virus itself may select strongly for viral evasion whereas the attack on the host pathways is less likely to elicit such responses(4). In this survey, we classify the studies by the life-cycle stage where the drug imposes its effects. The stages and publications are: attachment and entry(5-9), translation of polyproteins(10-13), proteolytic processing $(14,15)$, transcription and replication(16-18)and multiple stages(19, 20). It is generally accepted that comprehensive clinical benefits have not yet been demonstrated.

Supplementary Table 1 summarize the results of the survey. Given the complexities and variations of these experimental studies(5-23), we attempt to identify proposals that fulfill the following two criteria: 1) The application of the drug to the animal model results in the clearance of SARS-CoV-2 in $>80 \%$ of the animals within a reasonable time $(<$ one week). The clearance as shown by both the viral RNA measurement and the viral titer must be significantly faster than the control; and 2) The animals show no adverse effects at the end of the experiment. (In studies where the comparison is also made against remdesivir, we further require the performance to be no worse than that of remdesivir.)

In the summary of Supplementary Table 1, we did not find a published scheme that fulfills both criteria. We therefore aim to find a drug that can do so. Furthermore, it would be better if the drug is FDA-approved, readily available, inexpensive and amenable to largescale application.

\section{The general weakness of coronaviruses}

Before we identify a suitable drug, we shall first examine the weaknesses of the viruses. Coronaviruses possess the largest genomes (26.4 to $31.7 \mathrm{~kb}$ ) among all known RNA viruses and use two thirds of their genomes to make a super-peptide comprising $\sim 16$ non-structural proteins (NSPs), synthesized in concatenation and later proteolyzed into its component proteins (24). In SARS-CoV-2 and its relatives, the super-protein is $>700$ kilodaltons and $\sim 8000$ amino acids in size. Since human peptides are rarely larger than 5000 amino-acids long and the few large ones are often highly tissuespecific with a relatively long half-life, the viral super-protein stands out among the host's proteins.

The peculiar way of making a super-protein for later proteolysis has been hypothesized to be the Achilles heel of SARS-CoV-2(25). We may extend their arguments in two ways. First, a drug that blocks the initiation of the translation can be highly effective against the virus since one blockage can abrogate all 16 NSPs. Second, if a drug works to block the elongation, it would be possible to fine-tune the 
dosage to block peptide translation of $>5000$ amino acids, but much less so for smaller peptides.

In addition to the protein size, it has been pointed out that a common weakness of virus-infected cells is hyper-transcription or hyper-translation activities. After all, viruses often replicate at an exceedingly high rate. The heightened activities are true in cancer cells as well (25) and that may be why many anti-cancer and anti-virus drugs often target the transcription or translation machinery(26). It is hence possible that some drugs may be effective against both cancers and viruses. Generally, virusinfected cells divert up to $80 \%$ of the translation capacity to serve the unrelenting demand of the virus. The mechanism by which SARS-CoV-2 drives the heightened translation has recently been clarified(27).

\section{The specific mechanism of suppression by Homo-harringtonine (HHT)}

Homo-harringtonine (HHT), or omacetaxine mepesuccinate in its semi-synthetic form, is a cytotoxic plant alkaloid extracted from Cephalotaxus species and is likely the strongest inhibitor of protein translation approved for clinical use(28). HHT has been commonly used in China to treat cancer patients since the 70's. It is the first agent approved by FDA (USA, in 2012) targeting the mRNA translation process(29). Our survey(5-23)also found other drugs that target the cell's translation machinery for treating COVID-19(10-12), including Plitidepsin(10).

Given its wide use, the molecular mechanism of HHT has been well understood(30-32). HHT is described as a drug against peptide elongation (see Fig. 1A), especially "initial elongation" $(30,31)$. The latter may simply mean a highly efficient blockage at a high (and non-clinical) dose in vitro as HHT does not affect translation initiation. Based on the structural data of Garreau de Loubresse et al.(31), Fig. 1B shows graphically how HHT competes with the amino acid side chains of aminoacyl-tRNAs for binding to the A-site cleft of the ribosome.

Thanks to the highly specific mechanism of viral repression, the HHT scheme is expected to be effective at a very low dose. The efficacy, if true, would have the following advantage in the clinical application: if we target organs with the highest viral loads (say, the respiratory system) and deliver a low dose of HHT locally (by nebulization, for example), the treatment could be effective with minimal adverse effects. The toxicity is minimized due to the low drug concentration outside of the target area.

\section{The efficacy of HHT against coronaviruses}

Interestingly, since the SARS of 2003, there have been many efforts to identify drugs capable of repressing coronaviral replication. In each of these drug-screen 
studies(33-37), multiple drugs are reported to be effective against some coronaviruses. Curiously, there was not much emphasis that HHT (and only HHT) appears in almost all lists. Before 2020, five coronaviruses have been documented to be repressed by HHT(33-35)- MHV (murine coronavirus mouse hepatitis virus), Bovine coronavirus strain L9 (BCoV-L9), human enteric coronavirus strain 4408 (HECoV-4408), porcine epidemic diarrhea virus (PEDV) and MERS (Supplementary Table 2).

\section{Fig. 2 with 3 panels}

Because these reports are spread among laboratories using various dosages, the HHT effect might be due to diverse factors. To test the hypothesis of a common mechanism, we use PEDV from the previous list (34) and two additional porcine coronaviruses - SADS-CoV and PD-CoV in the same experimental setting. Our goal is to find out whether the dose-response among coronaviruses is indeed similarly low. Fig. 2 shows that the IC50 is generally around $100 \mathrm{nM}$ and the eradication is achieved at $<1 \mathrm{uM}$. We note that these are the same range of values reported in the literature $(13,33-37)$. In summary, eight different coronaviruses, including SARS$\mathrm{CoV}-2$, have been shown to be repressible by HHT at a comparable concentration. HHT thus appears to act against a general feature in protein translation.

\section{The efficacy of HHT in suppressing SARS-CoV-2 in mouse models}

We now present data on HHT efficacy against coronaviruses in vivo. The main experiments on infected mice are done in a $\mathrm{P} 4$ laboratory which uses a particular SARS-CoV-2 strain capable of infecting wildtype mice(38). HHT is delivered via intraperitoneal injection (IP), intranasal dripping (IN) or both (IP+IN). The IP part of the experiment was replicated in another P3 laboratory that uses the transgenic mice carrying the human ACE-2 receptor (39) and a common SARS-CoV-2 strain.

In the initial experiments, the main question was the efficacy of viral repression, leaving the toxicity issue for later resolution. Since $1 \mathrm{uM}$ concentration of HHT for 2 days can eradicate SARS-CoV-2 in vitro, we administered the drug at the theoretical concentration of $5 \mathrm{uM}$ on the first day and $2.5 \mathrm{uM}$ on each subsequent day, or roughly $80 \mathrm{ug}$ and $40 \mathrm{ug}$ per mouse per day (see Methods for the rationale). In Fig. 3A and 3B, HHT via IP infection appears highly effective in 3-4 days by either protocol. The viral load is not detectable in all but one case where the load is reduced to $<1 \%$. Hence, viral eradication is achievable in vivo as in the in vitro assays.

Fig. 3 and Fig. 4 here 
At the high concentration of $>1 \mathrm{uM}$, the efficacy as well as some adverse effects may both be expected. We interpret $5 \mathrm{uM}$ to be close to the maximal tolerated dose (MTD) for HHT in mice because even the slight variation in experimental conditions at this dose can result in fairly different outcomes (little toxicity in the experiments of Fig. 3B but substantial adverse effects in 3A). It is also interesting that a high dose near MTD for a short duration as in Fig. 3B could be effective with acceptable toxicity.

We then attempt to maintain the efficacy but avoid the toxicity. In a modified experiment that delivers the drug by nasal dripping only, the total dose is half of that in the IP+IN set. Most significantly, the efficacy in the IN experiment is the same as in the IP+IN experiment (Fig. 3A vs. Fig. 3C-3D) at half the total dose and, importantly, sans the adverse effects. In short, nasal dripping alone is fully effective in clearing the virus. The additional IP injection may be harmful without benefits.

\section{The safety dosage of HHT by nebulization}

While nasal dripping works in mice, it may not work in larger animals. We therefore carry out nebulization on dogs to measure the toxicity in larger mammals. At 10-12 kg, dogs should be more comparable with humans in toxicity tolerance although they do not get infected by SARS-CoV-2(40). Among animal models, dogs are exceptional in being able to cooperate to receive daily nebulization without anesthetization. The particles in nebulization, $\sim 5 \mathrm{um}$ in diameter, are expected to be able to reach the lungs. In our experiment, the dosage is $0 \mathrm{mg}, 0.5 \mathrm{mg}, 1 \mathrm{mg}$ and 1.5 $\mathrm{mg}$ per day for 7-10 days. By the end of the experiment, all dogs appear normal in body weight, blood cell count and blood biochemistry as shown in Fig. 4A-C. The dog receiving the highest dose was euthanized and the autopsy appears normal as well. The remaining dogs have been doing well. In short, dogs can tolerate HHT nebulization at doses much higher than the calculated dosage for treating human patients.

\section{Discussion}

HHT is effective in repressing all 8 coronaviruses tested in vitro(13, 33-37). The drug concentration necessary for viral clearance in vitro is consistently under $1 \mathrm{uM}$. (An anomaly in the literature is Choy et al. (2020)(41) which shows discrepancies with the literature in multiple drugs; see Wen et al. (2021)(13) and Ianevski et al. (2020)(37)) As stated above, the specific mechanism of HHT repression of viral protein translation may be the key.

For the treatment scheme to be ready for the next coronavirus epidemic in either humans or animals, an unresolved issue is the mode of delivering HHT. Although 
intravenous injection (I.V.) is the standard delivery method to treat leukemia and the safety level is well known(29, 42), I.V. is not necessarily the best option for treatment. I.P. injection in mice shows evidence of toxicity in one of the two experiments (Fig. 3A vs. 3B). Overall, the systemic administration is neither desirable nor necessary if the goal is to reduce the viral burden with minimal toxicity.

For the potential clinical applications of HHT on coronavirus patients, animal experiments point to nebulization. By nebulization, the drug is concentrated in the respiratory track, where the viral load is the highest(43). While the virus is known to invade other organs, the clearance from the respiratory track may allow the immune system to cope with the remaining viral loads. Using a portable device (like the one used for asthma), nebulization can be used on a large number of infected patients without hospital stay.

With the rapid developments of vaccines, the HHT treatment scheme should certainly be thought of as one for the future. As for the current epidemics, we have developed a protocol for a clinical trial in the Ditan hospital where all COVID-19 patients from the Beijing area were treated. In this protocol, we consider the merger of phase I and phase II trials. Given the rate of self-recovery with COVID-19 at $>95 \%$, most of the infections with no or light symptoms would be like healthy individuals recruited into the standard phase I trial. However, unlike healthy individuals in a phase I trial, a large number of infections with mild symptoms might benefit from the HHT application while providing the safety data, starting at the lowest doses of the standard phase I trial.

Since the protocol was approved by the IRB of Ditan in February of 2021, there have been no COVID-19 patients in or near Beijing. Until the vaccinations take full effects globally, many regions may still benefit from the HHT treatment scheme. We are therefore offering the protocol to certified medical facilities that might benefit from a clinical trial for treating COVID-19.

\section{Methods}

\section{Facility, Ethics, and Biosafety statement}

Main experiments with infectious SARS-CoV-2 were performed in the biosafety level 4 and animal biosafety level 4 facilities in the Harbin Veterinary Research Institute (HVRI) of the Chinese Academy of Agricultural Sciences (CAAS), approved by the Ministry of Agriculture and Rural Affairs of China.

Part of the in vivo antiviral studies were carried out at biosafety level-3 (BSL3) conditions at the Key Laboratory of Animal Models and Human Disease Mechanisms 
of the Chinese Academy of Sciences, Kunming Institute of Zoology (KIZ). The animal studies were carried out in strict accordance with the recommendations in the Guide for the Care and Use of Laboratory Animals of the Ministry of Science and Technology of the People's Republic of China. The protocols were approved by the Committee on the Ethics of Animal Experiments of the HVRI of CAAS or Institutional Committee for Animal Care and Biosafety at Kunming Institute of Zoology, Chinese Academy of Sciences, respectively. All animals used in this study were chosen randomly.

\section{Cells and Viruses at HVRI in Harbin}

Vero E6 cells were maintained in Dulbecco's modified Eagle's medium (DMEM) containing $10 \%$ fetal bovine serum (FBS) and antibiotics and incubated at $37^{\circ} \mathrm{C}$ with 5\% CO2. Mouse-adapted SARS-CoV-2/HRB26/human/2020/CHN (HRB26M, GISAID access no. EPI_ISL_459910) was obtained by serially passaging the HRB26 virus in 4-6-week-old female mice until passage 14 and was propagated in Vero E6 cells. Infectious virus titers were determined by using a plaque forming unit (PFU) assay in Vero E6 cells.

\section{Cells and Viruses at KIZ in Kunming}

The SARS-CoV-2 (strain 107) was provided by Guangdong Provincial Center for Disease Control and Prevention (Guangzhou, China). This virus was propagated and titrated on African green monkey kidney epithelial cells (Vero E6) (ATCC, no. 1586), which were cultured in Dulbecco's modified Eagle's medium (DMEM, Gibco) with 4.5 mM L-glutamine (GE Life Sciences) supplemented with 10\% FBS (Hyclone) and $1 \%$ penicillin-streptavidin (Gibco). Cells were cultured at $37^{\circ} \mathrm{C}$ in a humidified $5 \%$ $\mathrm{CO} 2$ atmosphere. Mycoplasma testing was performed at regular intervals and no mycoplasma contamination was detected.

\section{In vivo antiviral studies of HHT at HVRI in Harbin}

The mice for this study, 6-week-old female BALB/c, were obtained from Beijing Charles River Labs (Beijing, China). Mice were lightly anesthetized with $\mathrm{CO} 2$ and intranasally (I.N.) inoculated with $50 \mu \mathrm{L}$ dilutions of SARS-CoV-2. Body weights and clinical symptoms were monitored daily. Groups of six mice were treated by intraperitoneal (I.P.) injection with a loading dose of $80 \mathrm{ug} / \mathrm{mouse}$ HHT, followed by a daily maintenance dose of $40 \mathrm{ug} /$ mouse. In an allternative mode, mice were treated I.N. (40ug/mouse daily) alone or a combination of I.N. (40ug/mouse daily) and I.P. (40ug/mouse daily). As a control, mice were administered vehicle solution (PBS) daily. One hour after administration of the loading dose of HHT or vehicle solution, each mouse was inoculated i.n. with $10^{3.1}$ PFU of HRB26M in $50 \mu \mathrm{L}$. Three mice from each group were euthanized on days 3 and 5 post-inoculation. The nasal turbinates and lungs were collected for virus detection by qPCR and PFU assay $(38,44)$. 


\section{In vivo antiviral studies of HHT at KIZ in Kunming}

Experiments in KIZ were part of the multi-laboratory design but in a smaller scale than those in HVRI. The angiotensin-converting enzyme 2 (ACE2) humanized mice (hACE2 mice) aged 8-10 weeks were generated from Guangzhou Institute of Biomedicine and Health (GIBH), Chinese Academy of Sciences (CAS)(39). Mice were anesthetized with isoflurane (RWD Life Science, Shenzhen) and intranasally infected with $2 \times 10^{6}$ TCID 50 of SARS-CoV-2 (strain 107) in $30 \mu 1$ of DMEM. Body weights and clinical symptoms were monitored daily. Treated group of three mice were treated intraperitoneal (I.P.) with a loading dose of $80 \mathrm{ug} / \mathrm{mouse}$ HHT, followed by a daily maintenance dose of $40 \mathrm{ug} /$ mouse. As a control, mice $(n=3)$ were administered vehicle solution (PBS) daily. Lung tissues were collected on day 3 postinoculation. RNA was extracted from lung tissues using the TRIzolTM Reagent (Invitrogen) according to the manufacturer's instructions. Viral RNA was quantified by THUNDERBIRD ${ }^{\circledR}$ Probe One-step qRT-PCR Kit (Toyobo) according to the manufacturer's instructions and the TaqMan primers (Forward primer: 5' GGGGAACTTCTCCTGCTAGAAT-3'; Reverse primer: 5'CAGACATTTTGCTCTCAAGCTG-3'. The TaqMan probe sequences were 5'-FAMTTGCTGCTGCTTGACAGATT-TAMRA-3'.). The results were expressed as copies per microgram tissue.

\section{Evaluation of the anti-viral efficacy of HHT against other coronaviruses}

African green monkey kidney (Vero) cells were obtained from ATCC (ATCC number: CCL-81) (USA) and Porcine intestinal epithelial cell clone J2 (IPEC-J2) cells were obtained from Wen's Foodstuffs Group Co, Ltd (Guangdong, China). All cells were cultured in Dulbecco' s modified eagle medium (DMEM) (Hyclone, USA) supplemented with $100 \mathrm{U} / \mathrm{mL}$ penicillin, $100 \mathrm{U} / \mathrm{mL}$ streptomycin, and $10 \%$ fetal bovine serum (FBS) (BOVOGEN, Australia). The maintenance medium for PEDV, SADS-CoV, or PDCoV propagation was DMEM supplemented with $7.5 \mu \mathrm{g} / \mathrm{mL}$ trypsin (Gibco, USA)(45).

Confluent Vero or IPEC-J2 cell monolayers in 12-well plate were inoculated with various concentrations of HHT $(1-1000 \mathrm{nM})$ or the control normal DMEM for $1 \mathrm{~h}$, followed by infection with PEDV, SADS-CoV, or PDCoV at an MOI of 0.1 or 0.01 for $1 \mathrm{~h}$, and then the viral inoculums was removed and fresh maintenance medium containing different concentrations of HHT was added. Twenty-four hours later, cells were checked under microscopy to observe cytopathic effect (CPE) and then fixed for indirect immunofluorescent assay (IFA). Briefly, cells were fixed with $4 \%$ paraformaldehyde for $15 \mathrm{~min}$ and then permeabilized with $0.2 \%$ Triton $\mathrm{X}-100$ for 15 min at room temperature. After blocked with $1 \%$ bovine serum albumin (BSA), cells were stained with anti-PEDV (SADS-CoV, or PDCoV) N polyclonal antibody (Wen' $\mathrm{s}$ Foodstuffs Group Co., Ltd, China) $(1: 1000)$ at $37^{\circ} \mathrm{C}$ for $1 \mathrm{~h}$. Cells were then washed with $1 \times$ PBS and incubated with fluoresceinisothiocyanate (FITC) (1:500) or Cy3labeled goat anti-mouse secondary antibody (KPL, USA) $(1: 500)$ at $37^{\circ} \mathrm{C}$ for $1 \mathrm{~h}$. 
After three washes in $1 \times$ PBS, cells were counter-stained with DAPI and observed with a fluorescence microscope (LEICA DMi8, Germany).

\section{Evaluation the safety of HHT delivered by nebulization in a canine model}

Four beagles (female) weighing 10-12 kg were used in this study. The dogs were judged to be in good health based on the results of physical examinations, complete blood cell counts, and serum biochemical analyses. Each dog was fed with an appropriate amount of food and their health status was monitored daily by a dedicated veterinarian.

One dog was assigned to each of the following nebulization dosage: $0.5 \mathrm{mg} / \mathrm{day}$, $1.0 \mathrm{mg} /$ day, $1.5 \mathrm{mg} /$ day, or normal saline alone (control group). Dogs were treated for 7-10 days. Nebulization of HHT was performed in the animal research facility at the University of Kunming Medical University, using a commercially available ultrasonic nebulizer (particle size arounds 5 microns) connected to a polyethylene rebreathing bag. The polyethylene bag was held manually over the muzzle of the dog during treatments (15-20 minutes).

Blood samples were collected daily for complete blood cell counts and serum biochemical analyses. At the end of treatment, the dog received $1.5 \mathrm{mg}$ HHT per day was sacrificed and the lung tissue was collected for histopathological study. The other three dogs were adopted. 


\section{References}

1. "COVID-19 Weekly Epidemiological Update.," (World Health Organization., https://www.who.int/publications/m/item/weekly-epidemiological-update-oncovid-19---31-march-2021).

2. B. B. Oude Munnink et al., Transmission of SARS-CoV-2 on mink farms between humans and mink and back to humans. Science 371, 172 (2021).

3. S. Perlman, K. McIntosh, Coronaviruses, including severe acute respiratory syndrome (SARS) and Middle East respiratory syndrome (MERS). Mandell, Douglas, and Bennett's Principles and Practice of Infectious Diseases. 9th ed. Philadelphia, PA: Elsevier, (2020).

4. J. P. Wong, B. Damania, SARS-CoV-2 dependence on host pathways. Science 371, 884 (2021).

5. B. Hu, H. Guo, P. Zhou, Z.-L. Shi, Characteristics of SARS-CoV-2 and COVID-19. Nature Reviews Microbiology 19, 141-154 (2021).

6. M. Hoffmann et al., SARS-CoV-2 Cell Entry Depends on ACE2 and TMPRSS2 and Is Blocked by a Clinically Proven Protease Inhibitor. Cell 181, 271-280.e278 (2020).

7. A. C. Kalil et al., Baricitinib plus Remdesivir for Hospitalized Adults with Covid19. New England Journal of Medicine 384, 795-807 (2020).

8. M. Hoffmann et al., Camostat mesylate inhibits SARS-CoV-2 activation by TMPRSS2-related proteases and its metabolite GBPA exerts antiviral activity. EBioMedicine 65, (2021).

9. T. N. Hoang et al., Baricitinib treatment resolves lower-airway macrophage inflammation and neutrophil recruitment in SARS-CoV-2-infected rhesus macaques. Cell 184, 460-475.e421 (2021).

10. K. M. White et al., Plitidepsin has potent preclinical efficacy against SARS-CoV-2 by targeting the host protein eEF1A. Science 371, 926 (2021).

11. D. E. Gordon et al., A SARS-CoV-2 protein interaction map reveals targets for drug repurposing. Nature, (2020).

12. D. E. Gordon et al., Comparative host-coronavirus protein interaction networks reveal pan-viral disease mechanisms. Science 370, eabe9403 (2020).

13. H.-J. Wen et al., A proposal for clinical trials of COVID-19 treatment using homoharringtonine. National Science Review, (2021).

14. J. Qiao et al., SARS-CoV-2 M\&lt;sup\&gt;pro\&lt;/sup\&gt; inhibitors with antiviral activity in a transgenic mouse model. Science 371, 1374 (2021).

15. T. P. Sheahan et al., Comparative therapeutic efficacy of remdesivir and combination lopinavir, ritonavir, and interferon beta against MERS-CoV. Nature Communications 11, 222 (2020).

16. M. Wang et al., Remdesivir and chloroquine effectively inhibit the recently emerged novel coronavirus (2019-nCoV) in vitro. Cell Res 30, 269-271 (2020). 
17. B. N. Williamson et al., Clinical benefit of remdesivir in rhesus macaques infected with SARS-CoV-2. Nature 585, 273-276 (2020).

18. A. Wahl et al., SARS-CoV-2 infection is effectively treated and prevented by EIDD2801. Nature 591, 451-457 (2021).

19. S. Yuan et al., Metallodrug ranitidine bismuth citrate suppresses SARS-CoV-2 replication and relieves virus-associated pneumonia in Syrian hamsters. Nature Microbiology 5, 1439-1448 (2020).

20. S. Yuan et al., Clofazimine broadly inhibits coronaviruses including SARS-CoV-2. Nature, (2021).

21. N. Lian et al., Umifenovir treatment is not associated with improved outcomes in patients with coronavirus disease 2019: a retrospective study. Clinical Microbiology and Infection 26, 917-921 (2020).

22. L. Zhang et al., Comparative Antiviral Efficacy of Viral Protease Inhibitors against the Novel SARS-CoV-2 In Vitro. Virologica Sinica 35, 776-784 (2020).

23. T. P. Sheahan et al., An orally bioavailable broad-spectrum antiviral inhibits SARS-CoV-2 in human airway epithelial cell cultures and multiple coronaviruses in mice. Science Translational Medicine 12, eabb5883 (2020).

24. P. C. Woo, Y. Huang, S. K. Lau, K. Y. Yuen, Coronavirus genomics and bioinformatics analysis. Viruses 2, 1804-1820 (2010).

25. C.-I. $\mathrm{Wu}, \mathrm{H}$. Wen, Heightened protein-translation activities in mammalian cells and the disease/treatment implications. National Science Review, (2020).

26. T. Schneider-Poetsch, M. Yoshida, Along the Central Dogma-Controlling Gene Expression with Small Molecules. Annual Review of Biochemistry 87, 391-420 (2018).

27. S. Yuan et al., Nonstructural Protein 1 of SARS-CoV-2 Is a Potent Pathogenicity Factor Redirecting Host Protein Synthesis Machinery toward Viral RNA. Mol Cell 80, 1055-1066 e1056 (2020).

28. G. Cephalotaxus Research Coordinating, Cephalotaxine esters in the treatment of acute leukemia. A preliminary clinical assessment. Chin Med J 2, 263-272 (1976).

29. F. Alvandi et al., U.S. Food and Drug Administration approval summary: omacetaxine mepesuccinate as treatment for chronic myeloid leukemia. Oncologist 19, 94-99 (2014).

30. M. Fresno, A. Jiménez, D. Vázquez, Inhibition of translation in eukaryotic systems by harringtonine. Eur J Biochem 72, 323-330 (1977).

31. N. Garreau de Loubresse et al., Structural basis for the inhibition of the eukaryotic ribosome. Nature 513, 517-522 (2014).

32. G. Gürel, G. Blaha, P. B. Moore, T. A. Steitz, U2504 Determines the Species Specificity of the A-Site Cleft Antibiotics:: The Structures of Tiamulin, Homoharringtonine, and Bruceantin Bound to the Ribosome. Journal of Molecular Biology 389, 146-156 (2009). 
33. J. Cao, J. C. Forrest, X. Zhang, A screen of the NIH Clinical Collection small molecule library identifies potential anti-coronavirus drugs. Antiviral Res 114, 110 (2015).

34. H. J. Dong et al., The Natural Compound Homoharringtonine Presents Broad Antiviral Activity In Vitro and In Vivo. Viruses 10, (2018).

35. J. Dyall et al., Repurposing of clinically developed drugs for treatment of Middle East respiratory syndrome coronavirus infection. Antimicrob Agents Chemother 58, 4885-4893 (2014).

36. P. I. Andersen et al., Novel Antiviral Activities of Obatoclax, Emetine, Niclosamide, Brequinar, and Homoharringtonine. Viruses 11, (2019).

37. A. Ianevski et al., Potential Antiviral Options against SARS-CoV-2 Infection. Viruses 12, (2020).

38. J. Wang et al., Mouse-adapted SARS-CoV-2 replicates efficiently in the upper and lower respiratory tract of BALB/c and C57BL/6J mice. Protein Cell 11, 776-782 (2020).

39. F.-L. Liu et al., Rapid generation of ACE2 humanized inbred mouse model for COVID-19 with tetraploid complementation. National Science Review 8, (2021).

40. J. Shi et al., Susceptibility of ferrets, cats, dogs, and other domesticated animals to SARS-coronavirus 2. Science 368, 1016 (2020).

41. K. T. Choy et al., Remdesivir, lopinavir, emetine, and homoharringtonine inhibit SARS-CoV-2 replication in vitro. Antiviral Res 178, 104786 (2020).

42. X. Chen et al., Homoharringtonine is a safe and effective substitute for anthracyclines in children younger than 2 years old with acute myeloid leukemia. Frontiers of Medicine 13, 378-387 (2019).

43. R. Wölfel et al., Virological assessment of hospitalized patients with COVID-2019. Nature, (2020).

44. L. Shuai et al., Replication, pathogenicity, and transmission of SARS-CoV-2 in minks. National Science Review 8, (2021).

45. Z. Xu et al., Aloe extract inhibits porcine epidemic diarrhea virus in vitro and in vivo. Veterinary Microbiology 249, 108849 (2020). 


\section{Figure Legends}

Figure 1: The mechanism of HHT blockage of translation elongation, which underlies its anti-coronavirus efficacy. (A) HHT prevents the incoming aminoacyl-tRNA (A-tRNA) from unloading its amino acid cargo (P-tRNA) to extend the peptide by one amino acid; (B) Structural detail of the docking of HHT at the A site of the ribosome, resulting in the inhibition of translation elongation shown in 1A. The drawing is based on the structural data (PDB code: 4U4Q) in Garreau de Loubresse et al. (31).

Figure 2: HHT inhibits 3 different porcine coronaviruses in vitro at comparable doses $(<100 \mathrm{nM})$. While coronaviruses have been reported to be susceptible to HHT inhibition (33-37, and Supplementary Table 2), the test on additional coronaviruses here is to show comparable doses in the viral inhibition in the same experimental setting. If a general mechanism exists, comparable doses should be observed.

Figure 3: The repression of SARS-CoV-2 by HHT in mouse models. (A) I.P. (Intraperitoneal injection) or I.P.+I.N. (intranasal dripping) carried out in a P4 laboratory. The tissues assayed on the $4^{\text {th }}$ day after injection are given on the top. (B) The repeats of the I.P. experiment of (A) in another laboratory that uses a different mouse model and a different viral strain. The tissues assayed on the third day after injection are shown at the bottom. (C-D) I.N. only and the repression appears complete. Viral RNA and viral titer assays in the same P4 laboratory are presented. The horizontal dotted line (blue color) shows the detection limit of viral load in each assay. See the main text for details.

Figure 4: Evaluation the safety of HHT delivered by nebulization in a canine model. (A) Body weight changes during treatment. No adverse effects were detected. (B) complete blood cell count. (C) Biochemistry test. Values at (B) and (C) were standardized by $\mathrm{Z}_{\mathrm{i}, \mathrm{j}}=\left(\mathrm{x}_{\mathrm{i}, \mathrm{j}}-\mu_{\mathrm{j}}\right) / \sigma_{\mathrm{j}}$ of the normal distribution. Each dot is a sample, taken every day, from an experimental dog. For each test, three treated groups were compared with the control separately using Mann-Whitney U test and Bonferroni correction for multiple comparisons. The four measurements (amylase (AMY), cholesterol (CHOL), and glutamic-pyruvic transaminase (ALT) in the $0.5 \mathrm{mg} / \mathrm{day}$ group, and CHOL in the $1.5 \mathrm{mg} /$ day group) which were significantly different $(\mathrm{p}<0.01$ after correction for multiple comparisons) from the value in control group were marked by mocha arrows. Since all values in the four measurements were within the reference range, we concluded that these dosages administrated by nebulization were well-tolerated in dogs. 


\section{Figure 1}

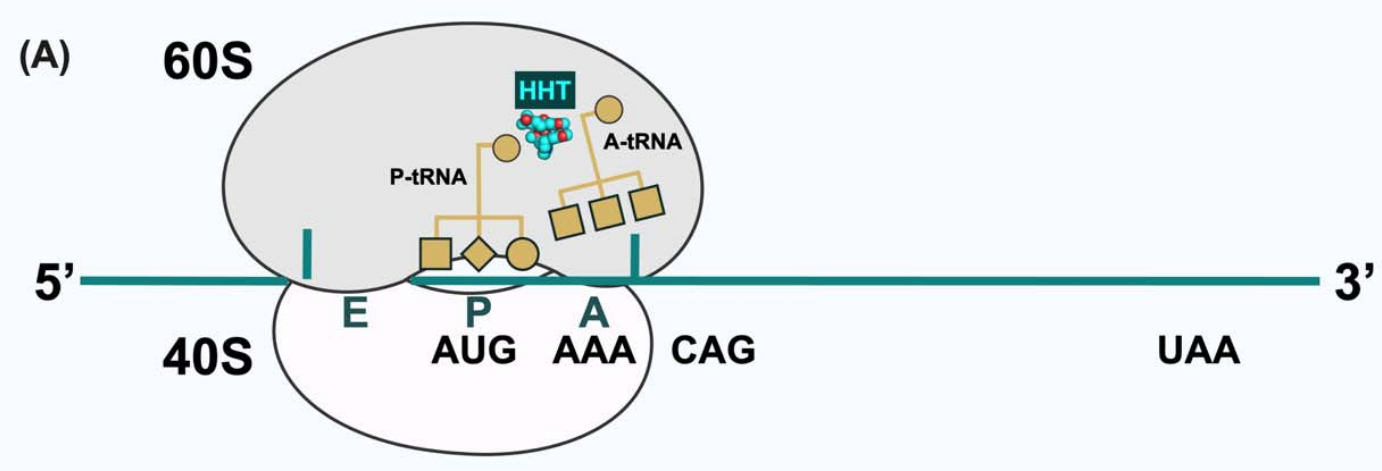

(B)

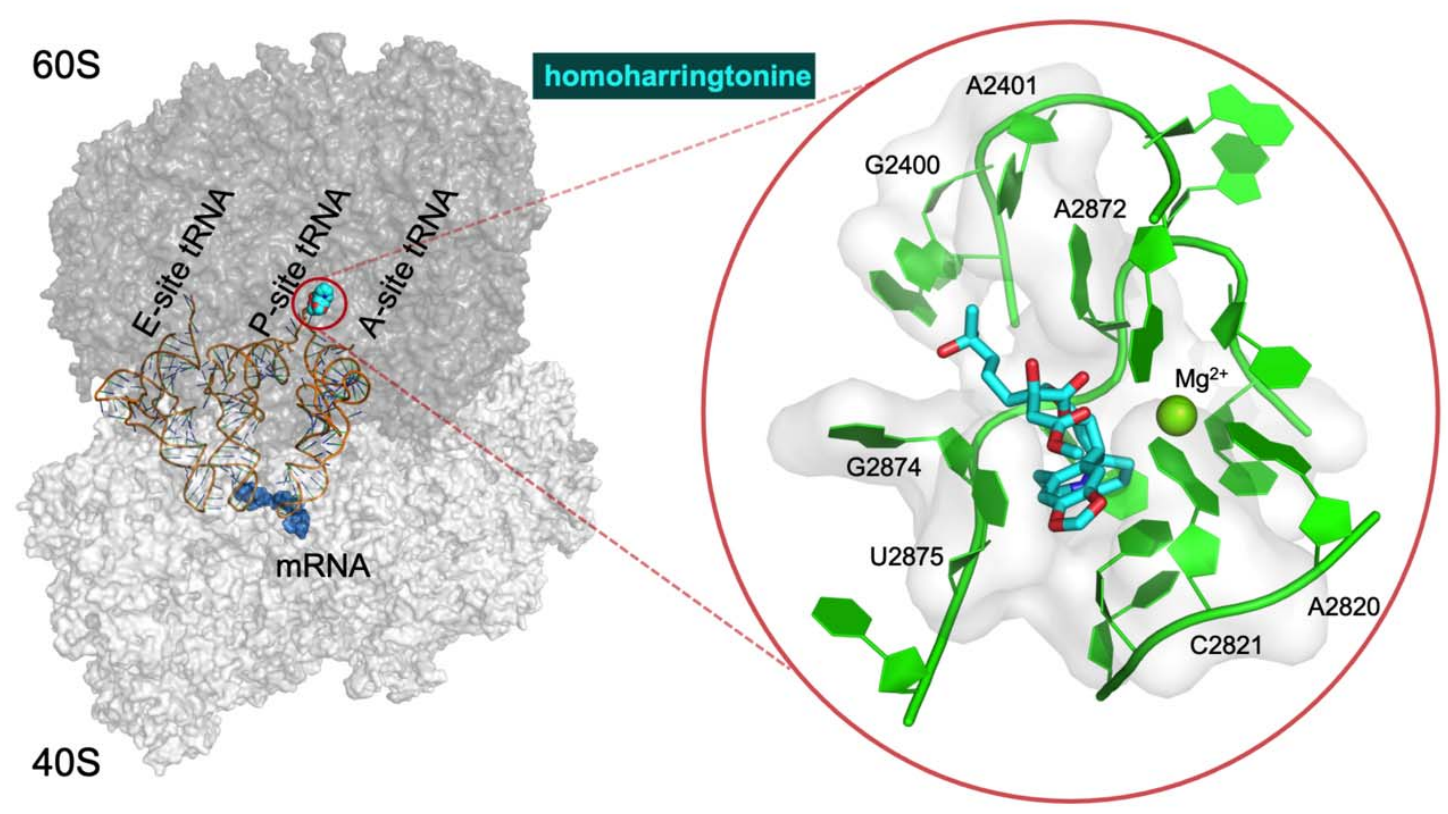


Figure 2

(A) 24 hours post $\mathrm{PEDV}$ infection $(\mathrm{MOI}=0.1)$

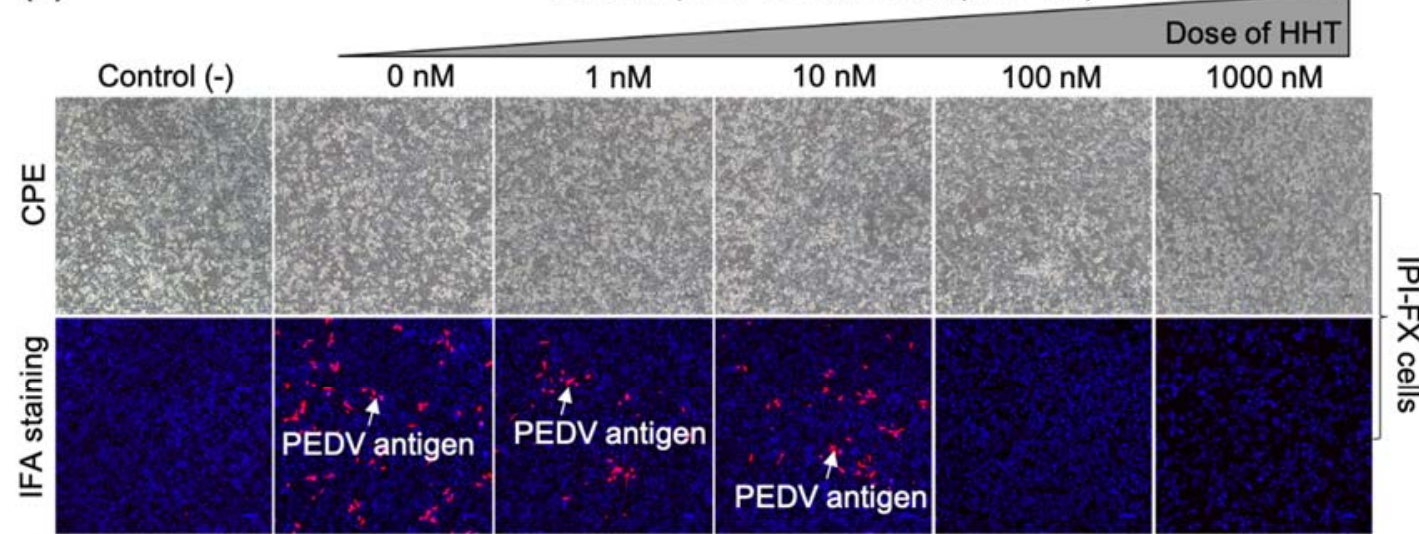

(B)

24 hours post SADS-CoV infection (MOI=0.1)

Dose of HHT

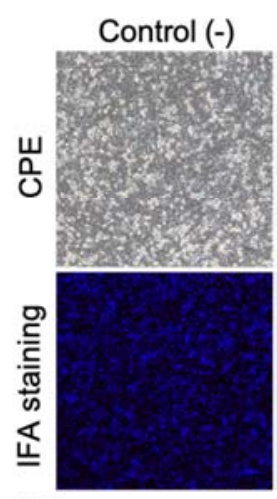

(C)

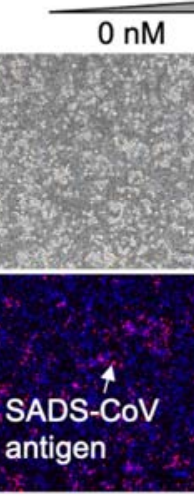

SADS-CoV

antigen

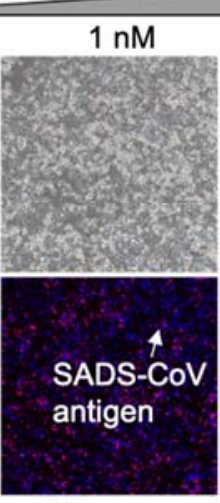

24 hours post $\mathrm{PDCoV}$ infection (MOI=0.01)
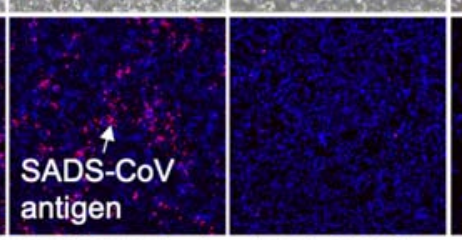
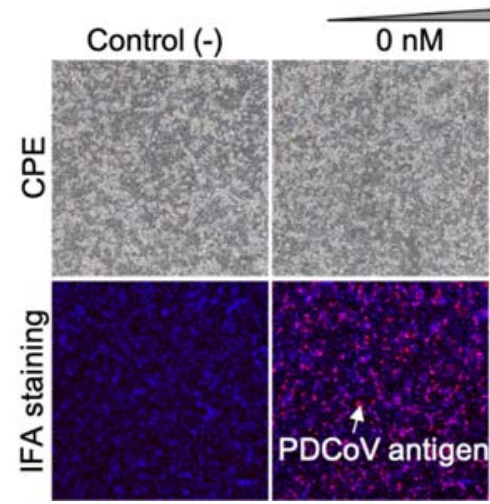

$1 \mathrm{nM}$

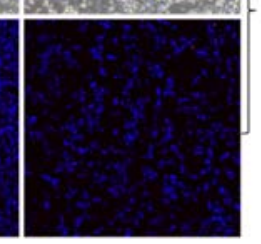

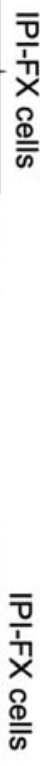

Dose of HHT
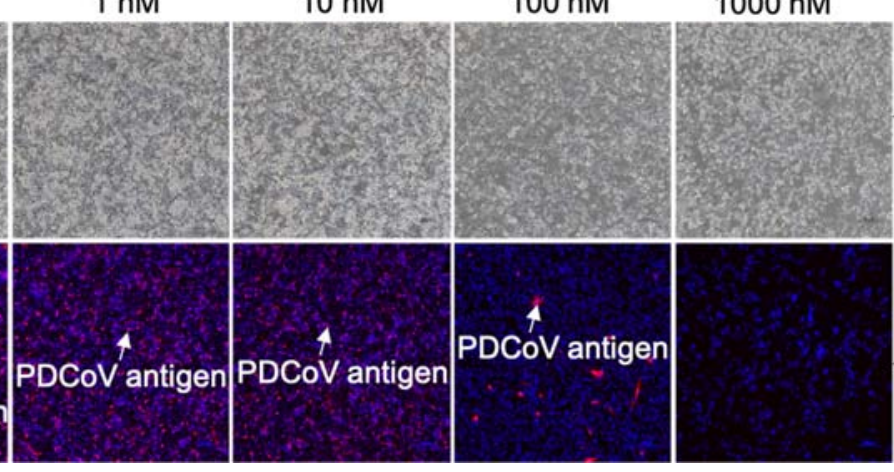


\section{Figure 3}

(A)

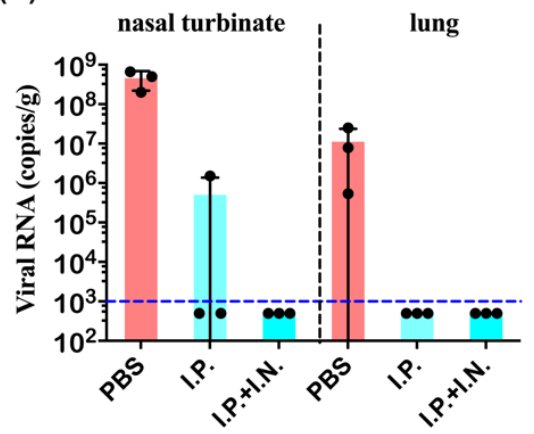

(C)

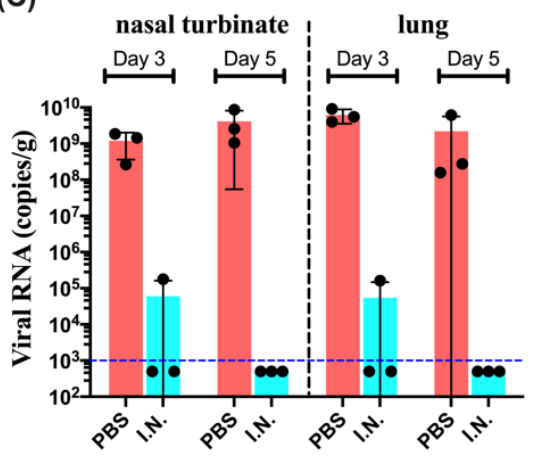

(B)

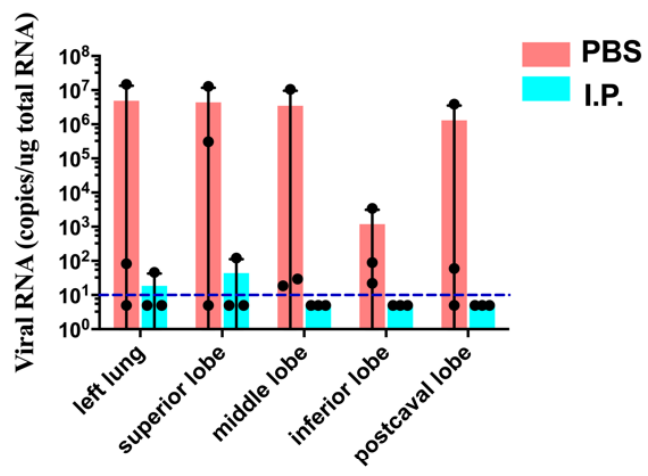

(D)

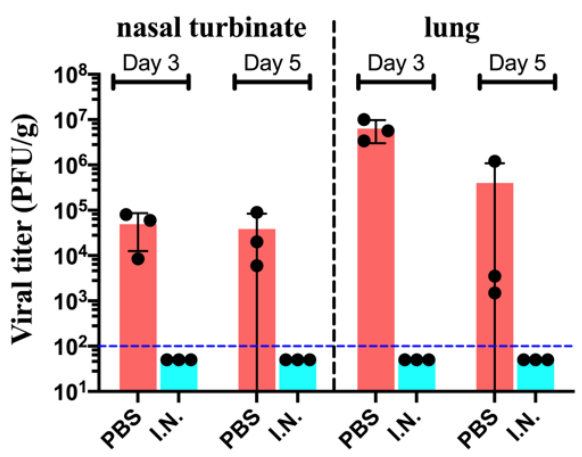


Figure 4

(A)
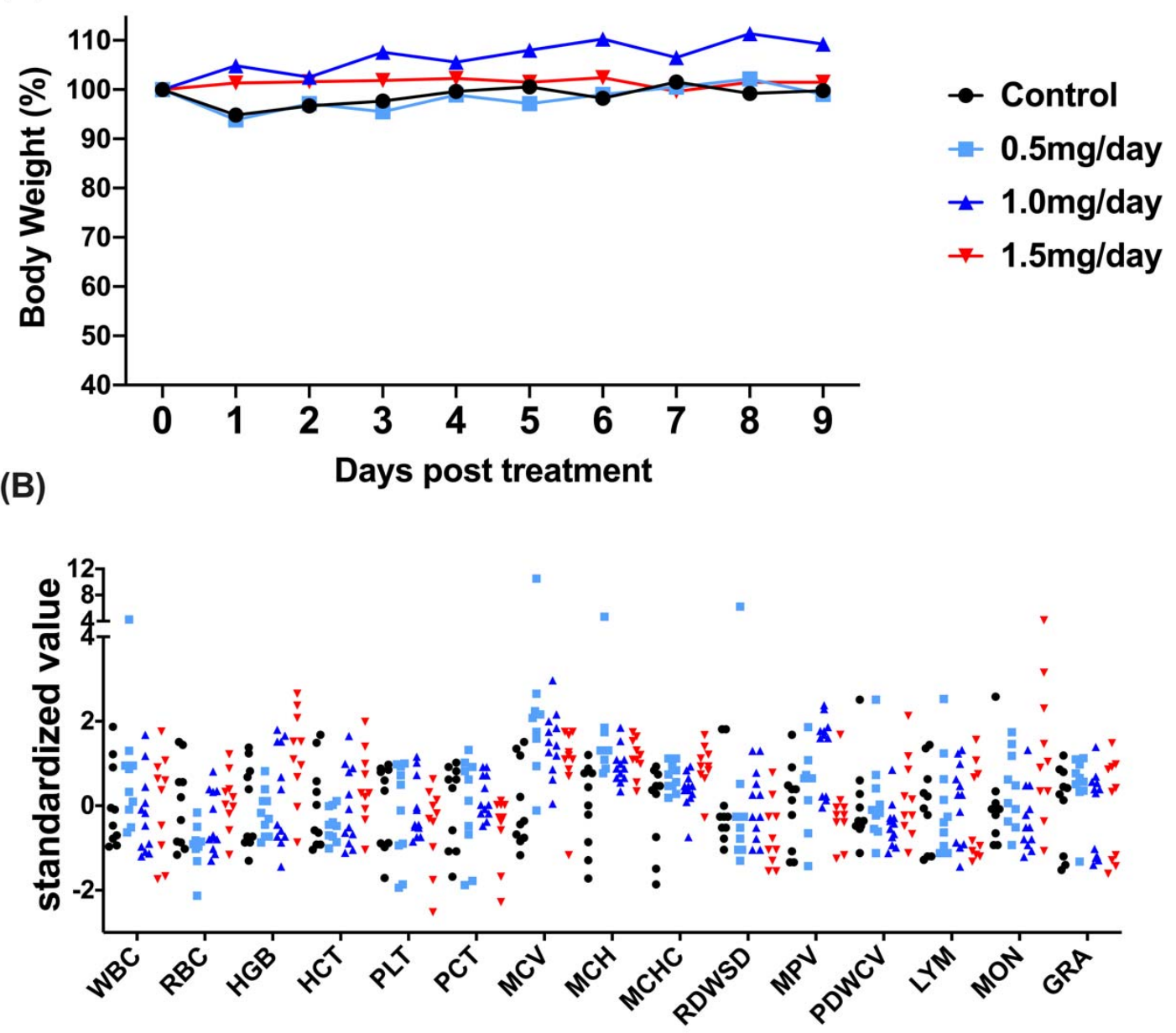

(C)

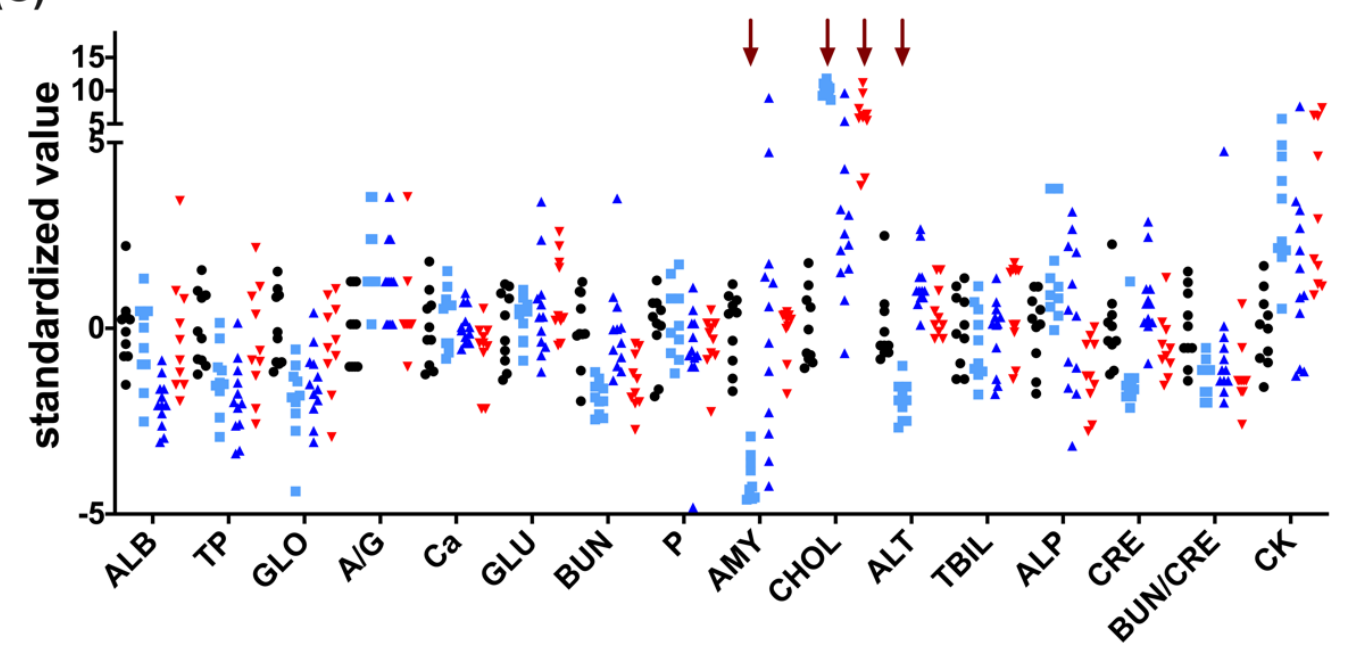


Supplementary Table 1. In vivo anti-SARS-CoV-2 efficacy of compounds targeting the viral life cycle.

\begin{tabular}{|c|c|c|c|c|c|}
\hline virus life cycle stage & Compound name & Primary indication & $\begin{array}{l}\text { Clinical Phase for } \\
\text { COVID-19 }\end{array}$ & $\begin{array}{l}\text { In vivo performance (compared to either } \\
\text { vehicle or Remdesivir) }\end{array}$ & References \\
\hline & & & & Remdesivir & \\
\hline \multirow[t]{3}{*}{ attachment and entry } & camostat mesilate & pancreatilis & Phase 2 & $\begin{array}{l}\text { about } 1 \text { log reduction in an } \\
\text { ex vivo model (PCLS) }\end{array}$ & $(1,2)$ \\
\hline & umifenovir & influenza & No clinical benefit & no in vivo data & (3) \\
\hline & baricitinib & rheumatoid arthritis & Phase 3 & no effect on virus replication & $(4,5)$ \\
\hline \multirow[t]{4}{*}{$\begin{array}{l}\text { initiation translation of } \\
\text { polyproteins }\end{array}$} & Plitidepsin & multiple myeloma & Phase $2 / 3$ & $\begin{array}{l}1.5-2 \log \text { reduction, } 4 / 8 \text { non-superior } \\
\text { achieved clearance in a } \\
\text { mouse model }\end{array}$ & $(6)$ \\
\hline & Ternatin-4 & $\begin{array}{l}\text { Preclinical } \\
\text { compound }\end{array}$ & & no in vivo data & $(7,8)$ \\
\hline & zotatifin & Phase 1 for cancer & Phase 1 & no in vivo data & $(7,8)$ \\
\hline & homoharritonine & leukemia & & 6/6 mice achieved & This study \\
\hline \multirow[t]{4}{*}{ proteolytic processing } & MI-09 & SARS-CoV-2 & animal model & $\begin{array}{l}\text { about } 2 \log \text { reduction, } \\
\text { clearance not achieved }\end{array}$ & (9) \\
\hline & MI-30 & SARS-CoV-2 & animal model & $\begin{array}{l}\text { about } 2 \log \text { reduction, } \\
\text { clearance not achieved }\end{array}$ & (9) \\
\hline & lopinavir & AIDS & No clinical benefit & no effect on virus replication & (10) \\
\hline & ritonavir & AIDS & No clinical benefit & no effect on virus replication & (10) \\
\hline $\begin{array}{l}\text { transcription \& RNA } \\
\text { replication }\end{array}$ & remdesivir & coronavirus & FDA-approved & $\begin{array}{l}\text { 10/36 rhesus macaques } \\
\text { achieved clearance on } 7 \mathrm{dpi}\end{array}$ & (11) \\
\hline
\end{tabular}




\begin{tabular}{|c|c|c|c|c|c|c|}
\hline & $\begin{array}{l}\text { EIDD-2801/MK- } \\
4482\end{array}$ & coronavirus & Phase 2a & $\begin{array}{l}5 \text { log reduction assayed by } \\
\text { PFU, } 4 / 8 \quad \text { achieved } \\
\text { clearance in the LoM model }\end{array}$ & & (12) \\
\hline \multirow[t]{2}{*}{ multiple steps } & clofazimine & leprosy & Phase 2 & $\begin{array}{l}\text { 1-2 log reduction in hamster } \\
\text { model, clearance not } \\
\text { achieved }\end{array}$ & less-effective & (13) \\
\hline & $\begin{array}{l}\text { ranitidine bismuth } \\
\text { citrate }\end{array}$ & anti-ulcer & & $\begin{array}{l}1-1.5 \text { log reduction, } \\
\text { clearance not achieved }\end{array}$ & non-superior & (14) \\
\hline
\end{tabular}


Supplementary Table 2. Homoharringtonine (HHT) exhibits broad-spectrum inhibition efficacy against coronaviruses.

\begin{tabular}{lllll}
\hline coronavirus & Abbreviation & Genus & IC50 (nM) & Reference \\
\hline Middle East respiratory syndrome coronavirus & MERS & Beta & 71.8 & Dyall et al. 2014(15) \\
\hline Mouse hepatitis coronavirus & MHV & Beta & 12 & Cao et al. 2015(16) \\
\hline Bovine coronavirus & BCoV & Beta & $<<1 \mathrm{uM}$ & Cao et al. 2015(16) \\
\hline Human enteric coronavirus & HECoV & Beta & $<<1 \mathrm{uM}$ & Cao et al. 2015(16) \\
Porcine epidemic diarrhea virus & PEDV & Alpha & 112 & Dong et al. 2018(17) \\
Severe acute respiratory syndrome coronavirus 2 & SARS-CoV-2 & Beta & $\sim 100$ & Wen et al. 2021(18) \\
\hline Severe acute respiratory syndrome coronavirus 2 & SARS-CoV-2 & Beta & 30 & lanevski et al. 2020(19) \\
Severe acute respiratory syndrome coronavirus 2 & SARS-CoV-2 & Beta & $2.1 \mathrm{uM}{ }^{\star *}$ & Choy et al. 2020(20) \\
\hline Porcine epidemic diarrhea virus & PEDV & Alpha & $<<100$ & This study \\
\hline Porcine deltacoronavirus & PDCoV & Delta & $<<100$ & This study \\
\hline Swine acute diarrhea syndrome coronavirus & SADS-CoV & Alpha & $<100$ & This study \\
\hline
\end{tabular}

** [Note - Choy et al.'s study reported a much larger IC50 value for multiple drugs, including HHT and Remdisivir (In Choy et al.'s study, IC50 of Remdisivir against SARS-CoV-2 was 26.9uM, while in many other studies, it was about $1 \mathrm{uM}(3,21))$.]

\section{References}

1. M. Hoffmann et al., Camostat mesylate inhibits SARS-CoV-2 activation by TMPRSS2-related proteases and its metabolite GBPA exerts antiviral activity. EBioMedicine 65, (2021).

2. M. Hoffmann et al., SARS-CoV-2 Cell Entry Depends on ACE2 and TMPRSS2 and Is Blocked by a Clinically Proven Protease Inhibitor. Cell 181,271

(2020/04/16/, 2020). 
3. A. Pizzorno et al., In vitro evaluation of antiviral activity of single and combined repurposable drugs against SARS-CoV-2. Antiviral Research 181,104878 $(2020 / 09 / 01 /, 2020)$

4. T. N. Hoang et al., Baricitinib treatment resolves lower-airway macrophage inflammation and neutrophil recruitment in SARS-CoV-2-infected rhesus macaques. Cel/ 184, 460 (2021).

5. A. C. Kalil et al., Baricitinib plus Remdesivir for Hospitalized Adults with Covid-19. New England Journal of Medicine 384, 795 (2021/03/04, 2020).

6. K. M. White et al., Plitidepsin has potent preclinical efficacy against SARS-CoV-2 by targeting the host protein eEF1A. Science 371, 926 (2021).

7. D. E. Gordon et al., A SARS-CoV-2 protein interaction map reveals targets for drug repurposing. Nature, (Apr 30, 2020).

8. D. E. Gordon et al., Comparative host-coronavirus protein interaction networks reveal pan-viral disease mechanisms. Science 370, eabe9403 (2020)

9. J. Qiao et al., SARS-CoV-2 M\&lt;sup\&gt;pro\&lt;/sup\&gt; inhibitors with antiviral activity in a transgenic mouse model. Science 371,1374 (2021).

10. T. P. Sheahan et al., Comparative therapeutic efficacy of remdesivir and combination lopinavir, ritonavir, and interferon beta against MERS-CoV. Nature Communications 11, $222(2020 / 01 / 10,2020)$.

11. B. N. Williamson et al., Clinical benefit of remdesivir in rhesus macaques infected with SARS-CoV-2. Nature 585, 273 (2020/09/01, 2020).

12. A. Wahl et al., SARS-CoV-2 infection is effectively treated and prevented by EIDD-2801. Nature 591, 451 (2021/03/01, 2021). 
13. S. Yuan et al., Clofazimine broadly inhibits coronaviruses including SARS-CoV-2. Nature, (2021/03/16, 2021).

14. S. Yuan et al., Metallodrug ranitidine bismuth citrate suppresses SARS-CoV-2 replication and relieves virus-associated pneumonia in Syrian hamsters. Nature Microbiology 5, 1439 (2020/11/01, 2020).

15. J. Dyall et al., Repurposing of clinically developed drugs for treatment of Middle East respiratory syndrome coronavirus infection. Antimicrob Agents Chemother 58, 4885 (Aug, 2014).

16. J. Cao, J. C. Forrest, X. Zhang, A screen of the NIH Clinical Collection small molecule library identifies potential anti-coronavirus drugs. Antiviral Res 114 , 1 (Feb, 2015).

17. H. J. Dong et al., The Natural Compound Homoharringtonine Presents Broad Antiviral Activity In Vitro and In Vivo. Viruses 10, (Nov 1, 2018).

18. H.-J. Wen et al., A proposal for clinical trials of COVID-19 treatment using homo-harringtonine. National Science Review, (2021).

19. A. lanevski et al., Potential Antiviral Options against SARS-CoV-2 Infection. Viruses 12, (Jun 13, 2020).

20. K. T. Choy et al., Remdesivir, lopinavir, emetine, and homoharringtonine inhibit SARS-CoV-2 replication in vitro. Antiviral Res 178, 104786 (Jun, 2020$).$

21. M. Wang et al., Remdesivir and chloroquine effectively inhibit the recently emerged novel coronavirus (2019-nCoV) in vitro. Cell Res 30, 269 (Mar, 2020). 